\title{
Collapsing Glomerulopathy
}

National Cancer Institute

\section{Source}

National Cancer Institute. Collapsing Glomerulopathy. NCI Thesaurus. Code C123044.

Segmental or global glomerulopathy with tuft wrinkling, collapse and contraction without increased matrix or cells, but with adjacent podocyte hypertrophy and hyperplasia. 